\title{
Sleep in Childhood Neurological Disorders
}

\author{
Sanjeev V. Kothare and Suresh Kotagal, editors; Demos Medical Publishing: New York, NY; \\ 439 p; ISBN: 978-1-933864-96-9; \$85 \\ Frank M. Ralls, M.D. ${ }^{1,3}$; Swala K. Abrams, M.D. ${ }^{2}$ \\ ${ }^{1}$ Division of Pulmonary, Critical Care, and Sleep Medicine, Department of Internal Medicine and ${ }^{2}$ Department of Psychiatry, \\ University of New Mexico School of Medicine, Albuquerque, NM; ${ }^{3}$ University of New Mexico Hospital Sleep Disorders Center, \\ Albuquerque, $N M$
}

S leep in Childhood Neurological Disorders is a rare book in that it captures one's interest on the very first page and sets a comfortable and unremitting pace until the final word of the final chapter. Each author has carefully chosen his/her subject material and succinctly summarizes the assigned topic such that the reader finds a finely tuned wealth of knowledge with something new in every chapter. Many of the commonly struggled with sleep disorders are addressed, defined, and then presented with material consistent with the latest literature on the subject. Disorders that may be common themes only to pediatric sleep specialists in tertiary care practices are also fully addressed, with key features of each disorder highlighted.

This book provides something for everyone who has an interest in pediatric sleep medicine, particularly for those whose practice includes the treatment of children with neurological pathology.

The first chapter immediately captivates the reader, beginning with the science of sleep, providing intriguing examples of the role of sleep in humans, reptiles, amphibians, birds, and other beings. The functions of sleep, sleep architecture, neuroanatomy, and neurotransmitters are clearly explained; this sets the stage for the ensuing chapters. Subsequent chapters provide practical ways to evaluate children with sleep disorders, examples of sleep histories, and discussion of other assessment tools.

The fundamental disorders of restless legs syndrome, childhood parasomnias, narcolepsy, and circadian rhythm disorders are clearly defined along with the pathogenesis, identification, treatments, and - most importantly for the purpose of this bookhow each disorder variably affects children with special needs.

Detailed attention is given to sleep in the context of epilepsy, neurodevelopmental disorders and children with brain tumors. Many readers will be surprised at the prevalence of seizures that occur primarily during sleep. In this book, information not readily offered or in a format not easily absorbed in other resources is served up like a delectable meal, ready to be tasted and savored. Topics include sleep and pediatric traumatic brain injury, craniofacial disorders, and Chiari malformations. The captivating subject of inverted melatonin secretion in disorders such as Smith-Magenis Syndrome is especially well done. Congenital central hypoventilation syndrome $(\mathrm{CCHC})$, which is eponymously referred to as Ondine's Curse, the etiologically defined gene PHOX2b, and ventilator management of these patients is expertly described.
The importance of the multidisciplinary team approach for evaluating children and psychiatric disorders is addressed, and there is a thorough explanation of how sleep problems adversely affect mood and neurobehavioral dysfunction. The authors do a nice job of discussing common psychiatric disorders and appropriate medication management.

Among our many favorite chapters was one describing the association of poor sleep and headaches, a subject that must be very familiar to all sleep specialists. Also discussed is the association of headaches and other sleep disorders, such as periodic limb movements of sleep, and treatment strategies are explored.

The chapter on pharmacology is like icing on a cake. The effects of various medications to induce sleep and the latest treatment of narcolepsy and obstructive sleep apnea are described in easy-to-understand and captivating detail.

The plethora of well-placed pictures, graphs, and summarizing paragraphs (including "pearls" and "key points") make this an especially welcome resource.

Sleep in Childhood Neurological Disorders will bring the reader up to date with the latest medical information on this subject, providing ideas which are immediately useful, as well as presenting new ideas that many will find fascinating and challenging.

In summary: Sleep in Childhood Neurological Disorders is a unique compilation of excellent chapters that brings much insight and understanding to the pathological sleep states that affect many children, especially those with neurological disorders. This book is interesting, captivating, and provides important up-to-date information. At only $\$ 85$, Sleep in Childhood Neurological Disorders is a must-read for any clinician who wishes to increase his/her understanding of sleep problems and have a positive impact in the pediatric population with neurological disorders. A book of this caliber should be considered as part of the routine curriculum of any sleep fellowship that emphasizes training in pediatric sleep medicine. This book is so well written that even the non-specialist is able to read and understand crucial information to help serve the needs of those very special patients.

\section{CITATION}

Ralls FM; Abrams SK. Sleep in childhood neurological disorders. J Clin Sleep Med 2013;9(3):291-292. 
SUBMISSION \& CORRESPONDENCE INFORMATION

Submitted for publication January, 2013

Accepted for publication January, 2013

Frank M. Ralls, M.D., Assistant Professor of Internal Medicine, Medical Director, Adult Sleep Medicine Services and Program Director, Fellowship in Sleep Medicine, University of New Mexico Hospital Sleep Disorders Center, 1101 Medical Arts Avenue NE, Building \#2, Albuquerque MM 87102; Tel: (505) 272-6110; Fax: (505) 272-6112;

E-mail: fralls@salud.unm.edu

\section{DISCLOSURE STATEMENT}

The authors have indicated no financial conflicts of interest. 